\title{
Effect of the pseudogap on the transition temperature in the cuprates and implications for its origin
}

\author{
Vivek Mishra ${ }^{1}$, U. Chatterjee ${ }^{2}$, J. C. Campuzano ${ }^{1,3}$ and M. R. Norman ${ }^{1 \star}$
}

Cuprates possess a large pseudogap that spans much of their phase diagram ${ }^{1,2}$. The origin of this pseudogap is as debated as the mechanism for high-temperature superconductivity. In one class of theories, the pseudogap arises from some instability not related to pairing, typically charge, spin or orbital current ordering. Evidence of this has come from a variety of measurements indicating symmetry breaking ${ }^{3-6}$. On the other side are theories where the pseudogap is associated with pairing. This ranges from preformed pairs ${ }^{7}$ to resonating valence bond theories where spin singlets become charge coherent ${ }^{8}$. Here, we study pairing in the cuprates by constructing the pair vertex using spectral functions derived from angle-resolved photoemission data. Assuming that the pseudogap is not due to pairing, we find that the superconducting instability is strongly suppressed, in stark contrast to what is actually observed. We trace this suppression to the destruction of the BCS logarithmic singularity from a combination of the pseudogap and lifetime broadening. Our findings strongly support those theories of the cuprates where the pseudogap is instead due to pairing.

To construct the pair vertex, we must first know the singleparticle Green's function. An issue is that angle-resolved photoemission spectroscopy (ARPES) measures only occupied states. As in earlier work, we surmount this difficulty under the assumption of particle-hole symmetry with respect to the Fermi energy and Fermi surface $\left(k_{\mathrm{F}}\right)$

$$
A(k, \omega)=I(k, \omega)+I\left(-k+2 k_{\mathrm{F}},-\omega\right)
$$

where $I$ is the photoemission intensity and $\omega$ is measured relative to the chemical potential. Relaxing this approximation should lead to only quantitative differences in the results (particle-hole asymmetry will act to suppress pairing). The assumption that the left-hand side of the equation can be equated to the spectral function (imaginary part of the single-particle Green's function) requires subtracting any background from the intensity (obtained from data for unoccupied momenta well beyond $k_{\mathrm{F}}$ ), and then normalizing by requiring the integrated weight over frequency to be equal to unity. A similar method has been successfully employed by us in several works, most recently ${ }^{9}$ to construct the dynamic susceptibility in cuprates, which was found to be in good agreement with inelastic neutron scattering (INS) data. In fact, the data set we employ here, from a near-optimal doped $\mathrm{Bi}_{2} \mathrm{Sr}_{2} \mathrm{CaCu}_{2} \mathrm{O}_{8+\delta}$ sample with a $T_{c}$ of $90 \mathrm{~K}$, was used in that work to reproduce the momentum and energy dependence of the INS data in the superconducting state, in particular the unique hourglass-like dispersion observed in a variety of cuprates. In our case, though, we will use normal-state data above $T_{\mathrm{c}}$. For this sample, a relatively complete momentum sweep was done in an octant of the Brillouin zone at a temperature of $140 \mathrm{~K}$ (ref. 10) and exhibits a pronounced pseudogap with a gapless arc. The data were obtained on a $2 \mathrm{meV}$ energy grid down to $322 \mathrm{meV}$ below the Fermi energy, with the background intensity adjusted to match each spectrum at this lower energy cutoff.

To proceed, we will assume that pairing originates from electronelectron interactions. Although the particular approach used here is based on spin fluctuations, we believe the results are general to any electronic pairing mechanism. This first requires constructing the polarization bubble

$$
\begin{array}{r}
\chi_{0}(q, \Omega)=\int_{-\infty}^{\infty} \mathrm{d} \omega \int_{-\infty}^{\infty} \mathrm{d} \omega^{\prime} \frac{f(\omega)-f\left(\omega^{\prime}\right)}{\omega-\omega^{\prime}+\Omega+i 0^{+}} \\
\times \frac{1}{N} \sum_{k} A(k+q, \omega) A\left(k, \omega^{\prime}\right)
\end{array}
$$

where $f(\omega)$ is the Fermi function and $N$ is the number of $k$ points. The $k$ sum is restricted to two dimensions under the further assumption of a single band (for the data set we use, there is no evidence for bilayer splitting). We will then make the standard random phase approximation to construct the full dynamic susceptibility

$$
\chi(k, \Omega)=\frac{\chi_{0}(k, \Omega)}{1-U \chi_{0}(k, \Omega)}
$$

where $U$ is an effective screened Hubbard interaction appropriate for a single band involving hybridized copper $3 d$ and oxygen $2 p$ orbitals.

In Fig. 1, we show the imaginary part of $\chi$ for two different values of $U$ along the $(0,0)-(\pi, \pi)$ direction. For the larger value of $U(860 \mathrm{meV}), \operatorname{Im} \chi$ is concentrated at low frequencies at the commensurate wavevector $(\pi, \pi)$. This is typical of very underdoped samples near the commensurate antiferromagnetic phase $^{11}$. We contrast this with a smaller value of $U(800 \mathrm{meV})$, where spectral weight is now concentrated at incommensurate wavevectors at a higher energy, being a more appropriate description of INS data $^{12}$ for slightly underdoped samples (consistent with the ARPES data set employed). Decreasing $U$ even further reduces the magnitude of $\operatorname{Im} \chi$, moves the incommensurate weight to even higher energies, and suppresses the lower energy commensurate weight. 
a
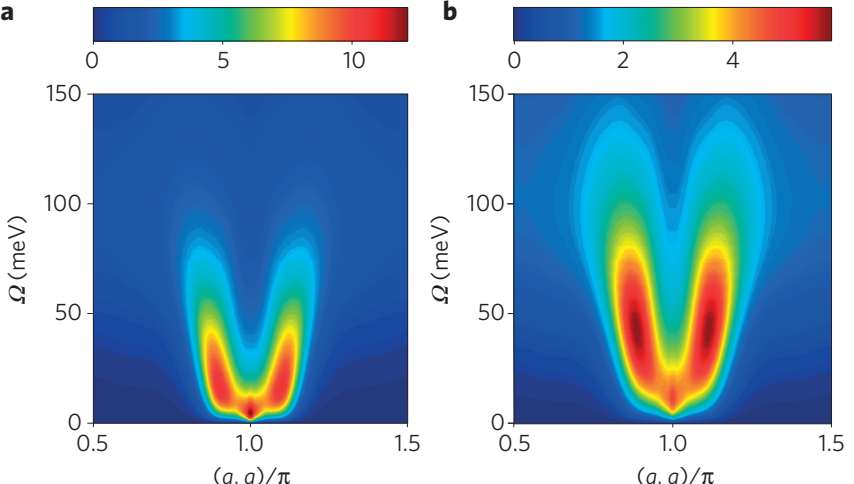

Figure $\mathbf{1}$ | Dynamic spin susceptibility. a,b, Imaginary part of the susceptibility $\chi^{\prime \prime}$ constructed from experimental Green's functions versus energy along the nodal direction for $U=860 \mathrm{meV}$ (a) and $U=800 \mathrm{meV}$

(b), where $U$ is an effective screened Hubbard interaction.

Using this $\chi$, the resulting electron-electron interaction is ${ }^{13,14}$

$$
V(k, \Omega)=\bar{U}^{2}\left[\frac{3}{2} \chi(k, \Omega)-\frac{1}{2} \chi_{0}(k, \Omega)\right]
$$

where $\bar{U}$ can differ from $U$ because of vertex corrections ${ }^{15}$. To set $\bar{U}$, we will require that the renormalized Fermi velocity at the $d$-wave node (the Fermi surface along the $(0,0)-(\pi, \pi)$ direction) matches that determined from the ARPES dispersion $(1.6 \mathrm{eV} \AA)$ assuming a bare velocity of $3 \mathrm{eV} \AA$ from band theory. The renormalization factor $(3 / 1.6)$ can be obtained as

$$
Z=\left[1-\frac{\partial \Sigma^{\prime}}{\partial \omega}\right]_{\omega=0}
$$

where $\Sigma^{\prime}$ is the real part of the fermion self-energy, and we assume $Z$ arises from the same interaction $V$ as above:

$$
\Sigma\left(k, i \omega_{n}\right)=T \sum_{q, \omega_{m}} V\left(k-q, i \omega_{n}-i \omega_{m}\right) G_{0}\left(q, i \omega_{m}\right)
$$

where $G_{0}$ is the bare fermion Green's function

$$
G_{0}^{-1}\left(k, i \omega_{n}\right)=i \omega_{n}-\xi_{k}
$$

and $\xi_{k}$ is the bare dispersion (obtained from a tight-binding fit to the ARPES dispersion by multiplying by the renormalization factor $3 / 1.6$ mentioned above). The real part of $\Sigma$ used to obtain $Z$ is obtained from the real frequency version of equation (6). For the case shown in Fig. 1a, $\bar{U}$ perchance turns out to be the same as $U$. However, for the case shown in Fig. 1b, we must increase $\bar{U}$ to $928 \mathrm{meV}$ to obtain the same $Z$ as for the first case.

We now turn to the pairing problem. The anomalous (pairing) self-energy in the singlet channel is ${ }^{13,14}$

$$
-\frac{T}{N} \sum_{k^{\prime}, \omega_{m}} V\left(k-k^{\prime}, i \omega_{n}-i \omega_{m}\right) \mathcal{P}_{0}\left(k^{\prime}, i \omega_{m}\right) \Phi\left(k^{\prime}, i \omega_{m}\right)=\Phi\left(k, i \omega_{n}\right)
$$

with the pairing kernel $\mathcal{P}_{0}$

$$
\mathcal{P}_{0}\left(k^{\prime}, i \omega_{m}\right)=G\left(k^{\prime}, i \omega_{m}\right) G\left(-k^{\prime},-i \omega_{m}\right)
$$

and $G$ is the fully dressed Green's function, which is formally determined by including the self-energy correction equation (6) in a completely self-consistent approach. Instead, we obtain $G$ from the experimental spectral functions. This is related to the approach of ref. 16 where INS data were used instead.

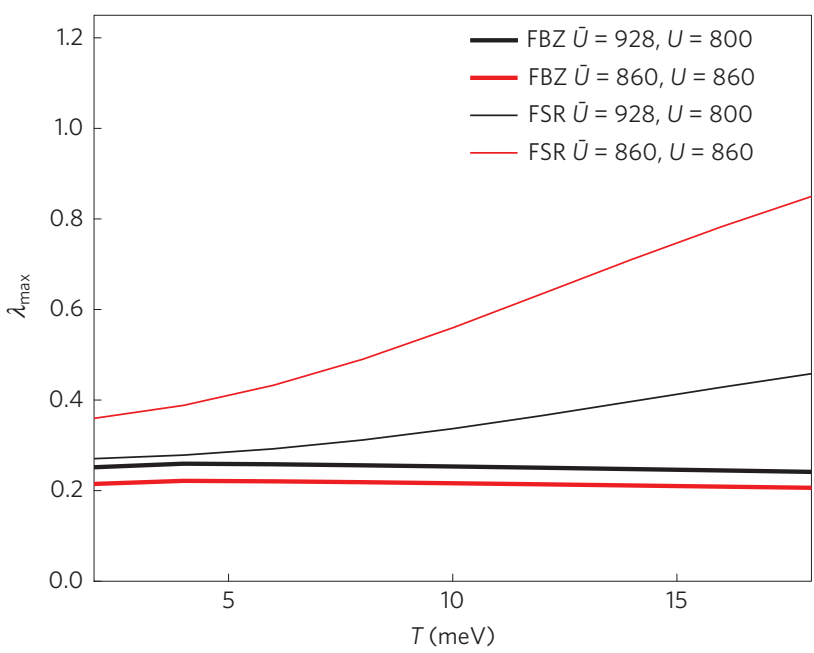

Figure $\mathbf{2}$ | No superconductivity in the presence of a non-pairing pseudogap. The leading $d$-wave eigenvalue $\lambda_{\max }$ as a function of temperature, using experimental spectral functions. FSR (thin curves) are from Fermi-surface-restricted calculations obtained from equation (10); FBZ (thick curves) are from full Brillouin zone calculations from equation (8). The interaction parameters $U$ and $\bar{U}$ are indicated in units of millielecronvolts. Note that $\lambda_{\max }$ is less than unity (that is, no solution to the superconducting gap equation).

At $T_{c}$, the maximum eigenvalue $\left(\lambda_{\max }\right)$ of equation (8) reaches unity and the corresponding eigenvector gives the energymomentum structure of the superconducting order parameter. Ideally, we would need to know $G$ at each temperature. This is impractical when using real experimental data. Instead, we use our experimental normal-state data at $140 \mathrm{~K}$, and assume that all temperature dependence arises from the Matsubara frequencies. As we will see below, this is a best case scenario, because if anything, the magnitude of the pseudogap increases as the temperature is lowered.

Our results are shown in Fig. 2, labelled as FBZ. We see that $\lambda_{\max }$ (which occurs for $B_{1 g}$, that is, $d$-wave, symmetry) is much less than unity and essentially temperature independent for both cases shown in Fig. 1. This implies that there is no superconductivity. This is the central result of our paper.

To understand this surprising result, we now turn to Fermisurface-restricted calculations, which approximate the full Brillouin zone results, and have the advantage that analytic calculations are possible. In this commonly employed approximation, the momentum perpendicular to the Fermi surface $\left(k_{\perp}\right)$ is integrated out with the dependence of $V$ on $k_{\perp}$ ignored. This procedure results in an equation that depends only on the angular variation around the Fermi surface:

$$
-\frac{T}{N_{\phi}} \sum_{\phi^{\prime}, \omega_{m}} V_{n m}^{\phi \phi^{\prime}} \mathcal{P}_{0}\left(\phi^{\prime}, i \omega_{m}\right) \Phi\left(\phi^{\prime}, i \omega_{m}\right)=\Phi\left(\phi, i \omega_{n}\right)
$$

where $N_{\phi}$ is the number of angular points and $V_{n m}^{\phi \phi^{\prime}}$ is

$$
V_{n m}^{\phi \phi^{\prime}}=V\left(k_{\mathrm{F} x}^{\phi}-k_{\mathrm{F} x}^{\phi^{\prime}}, k_{\mathrm{F} y}^{\phi}-k_{\mathrm{F} y}^{\phi^{\prime}}, i \omega_{n}-i \omega_{m}\right)
$$

$\mathcal{P}_{0}$ is obtained by numerically integrating equation (9) using the experimental $G$ over $k_{\perp}$, with the integration direction for each angle $\phi$ determined from the normal given by the tight-binding fit to the ARPES data (this same procedure is used to identify $k_{\mathrm{F}}$ in equation (1)).

The results are also shown in Fig. 2. Although $\lambda_{\max }$ is now temperature dependent, over the temperature range shown, it is still below unity. Paradoxically, $\lambda_{\max }$ increases with increasing 
temperature. We have verified that at even higher temperatures, $\lambda_{\max }$ reaches a maximum, and then begins to fall, with the more realistic second case $(U=800 \mathrm{meV})$ always remaining below unity. Similar behaviour for the $T$ dependence of $\lambda$ was reported in ref. 17 where a Mott gap is present.

To understand this behaviour, we now turn to some analytic calculations. To a good approximation, we can approximate $V$ for the $d$-wave case in the weak-coupling BCS limit as

$$
V\left(\phi, \phi^{\prime}\right)=\mathcal{V} \cos (2 \phi) \cos \left(2 \phi^{\prime}\right)
$$

and assume an isotropic density of states $N_{0}$ over the Fermi surface coming from $\xi_{k}$. The weak-coupling equation for $T_{\mathrm{c}}$ is

$$
T \sum_{\omega_{n}} \int_{0}^{2 \pi} \frac{\mathrm{d} \phi}{2 \pi} \mathcal{V} \cos ^{2}(2 \phi) P_{0}\left(\phi, i \omega_{n}\right)=1
$$

For $G$ we use a phenomenological form that is a good representation of ARPES data ${ }^{18}$

$$
G\left(k, i \omega_{n}\right)=-\frac{i \omega_{n}+i \Gamma \operatorname{sgn}\left(\omega_{n}\right)+\xi_{k}}{\left(\omega_{n}+\Gamma \operatorname{sgn}\left(\omega_{n}\right)\right)^{2}+\xi_{k}^{2}+\Delta_{k}^{2}}
$$

Here $\Gamma$ is the broadening and $\Delta_{k}$ is the anisotropic pseudogap, which, consistent with ARPES, is assumed to have a $d$-wave anisotropy. On the Fermi surface, this can be approximated as $\Delta_{0} \cos (2 \phi)$. The pairing kernel can now be analytically derived

$$
\mathcal{P}_{0}\left(\phi, i \omega_{n}\right)=\pi N_{0}\left[\frac{1}{\sqrt{\tilde{\omega}_{n}^{2}+\Delta_{\phi}^{2}}}-\frac{\Delta_{\phi}^{2}}{2\left(\tilde{\omega}_{n}^{2}+\Delta_{\phi}^{2}\right)^{3 / 2}}\right]
$$

Here $\tilde{\omega}_{n}$ is $\omega_{n}+\operatorname{sgn}\left(\omega_{n}\right) \Gamma$. To obtain an analytic approximation, we replace the sum $T \sum_{\omega_{n}}$ by an integral $\int \mathrm{d} \omega / 2 \pi$, using the EulerMaclaurin formula ${ }^{19}$ for low temperatures in equation (13) and rewrite the condition for $T_{\mathrm{c}}$ as

$$
1=N_{0} \mathcal{V} \int_{\pi T}^{\infty} \mathrm{d} \omega \int_{0}^{2 \pi} \frac{\mathrm{d} \phi}{2 \pi} \cos ^{2} 2 \phi\left[\frac{1}{\sqrt{\tilde{\omega}^{2}+\Delta_{\phi}^{2}}}-\frac{\Delta_{\phi}^{2}}{2\left(\tilde{\omega}^{2}+\Delta_{\phi}^{2}\right)^{3 / 2}}\right]
$$

The integral over $\omega$ can be carried out analytically. The second term is convergent, so we can integrate it to $\infty$. For the first term, we use a BCS cutoff energy $\omega_{\mathrm{c}}$ and we assume $\omega_{\mathrm{c}} \gg T, \Delta_{0}, \Gamma$ and in the low- $T$ limit we get

$$
\begin{gathered}
1 \simeq N_{0} \mathcal{V} \int_{0}^{2 \pi} \frac{\mathrm{d} \phi}{2 \pi} \cos ^{2} 2 \phi\left[\log \left(\frac{1}{\sqrt{\mathrm{e}}} \frac{2 \omega_{c}}{\Gamma+\pi T+\sqrt{(\Gamma+\pi T)^{2}+\Delta_{\phi}^{2}}}\right)\right. \\
\left.+\frac{\Gamma+\pi T}{2 \sqrt{(\Gamma+\pi T)^{2}+\Delta_{\phi}^{2}}}\right]
\end{gathered}
$$

By examining equation (17), we can clearly see that the logarithmic divergence of the first term is cutoff by both $\Gamma$ and $\Delta_{0}$, so a solution is no longer guaranteed. We can estimate the critical values of the inverse lifetime and pseudogap to kill superconductivity at $T=0$ for limiting cases. In the clean limit with $\Gamma=0, \Delta_{\text {cri }}=2 \pi \mathrm{e}^{-(\gamma+1)} T_{\text {c } 0}$, where $\gamma$ is Euler's constant and $T_{c 0}$ is $T_{c}$ for $\Delta_{0}, \Gamma=0$. With no pseudogap, we find a critical inverse lifetime $\Gamma_{\text {cri }}$ of $\pi \mathrm{e}^{-\gamma} T_{\mathrm{c} 0} / 2$ $\left(\right.$ Abrikosov-Gor'kov $\left.{ }^{20}\right)$. Figure 3 shows the numerically evaluated left-hand side of equation (13) (denoted as $\lambda_{\max }^{\mathrm{wc}}$ ) as a function of temperature for various $\Delta_{0}$, with the variation of $T_{c}$ with $\Delta_{0}$ or $\Gamma$ shown in the inset. One clearly sees that the logarithmic divergence is cut off as $\Delta_{0}$ increases, leading to a maximum in $\lambda$

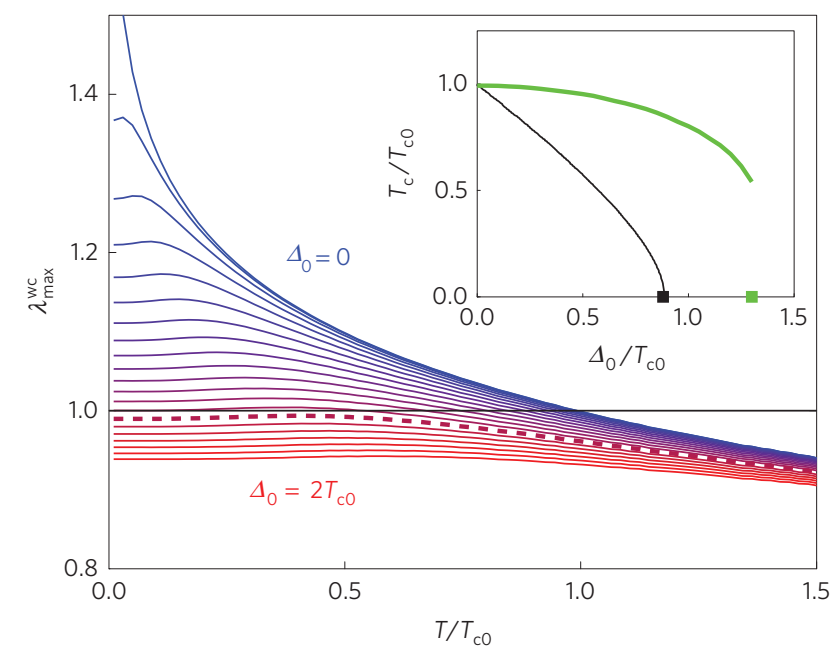

Figure 3 | Suppression of $T_{c}$ by a non-pairing pseudogap (weak coupling). $\lambda_{\max }^{\mathrm{wc}}$ for a $d$-wave superconductor with a $d$-wave pseudogap as a function of temperature plotted for various values of the pseudogap from 0 to $2 T_{\mathrm{c} 0}$. This quantity (left-hand side of equation (13), with $\Gamma=0$ ) is the weak-coupling analogue of $\lambda_{\max }$. All energies are normalized to the mean-field transition temperature $T_{\mathrm{co}}$ for $\Gamma, \Delta_{0}=0$. For large enough $\Delta_{0}$, a solution to the superconducting gap equation does not exist (dashed curve and ones below it). Inset: $T_{\mathrm{c}}$ as a function of the pseudogap. This thick green curve abruptly terminates when the maximum in $\lambda$ as a function of $T$ goes below unity. The thin black curve shows $T_{\mathrm{c}}$ as a function of the inverse lifetime $\Gamma$. In this case, the $x$ axis should be read as $\Gamma / T_{\mathrm{co}}$. The filled squares are the analytic estimates at $T=0$.

at a particular temperature. Once this maximum falls below unity, no superconducting solution exists.

To show that our findings are general and not limited to weakcoupling assumptions, we consider a calculation based on a $V$ derived from a phenomenological form for $\chi$ (refs 21,22):

$$
V(k, \Omega)=\frac{3}{2} g_{\mathrm{sf}}^{2} \frac{\chi_{\mathrm{Q}}}{\xi_{\mathrm{AF}}^{-2}+2+\cos k_{x}+\cos k_{y}-i \frac{\Omega}{\Omega_{\mathrm{sf}}}}
$$

where $g_{\text {sf }}$ is the coupling between fermions and spin fluctuations, $\xi_{\mathrm{AF}}$ is the antiferromagnetic coherence length, $\Omega_{\text {sf }}$ is the characteristic spin fluctuation energy scale, and $\chi_{\mathrm{Q}}$ is the static susceptibility at the commensurate vector $\mathbf{Q}=(\pi, \pi)$. For illustrative purposes, we take $g_{\text {sf }}^{2} \chi_{\mathrm{Q}}=0.27 \mathrm{eV}, \xi_{\mathrm{AF}}=10, \Omega_{\mathrm{sf}}=0.4 \mathrm{eV}$ with a cutoff energy for Im $\chi$ of $0.4 \mathrm{eV}$, although we have studied a variety of parameter sets (particularly variation of $\xi_{\mathrm{AF}}$ ). In general, these parameters are temperature dependent, but for simplicity we ignore this. We use the same model $G$ from above that was used to study the weak-coupling limit. Figure 4 shows the variation of $T_{c}$ with the pseudogap for different values of $\Gamma$. As in the weak coupling case, $\Delta_{0}$ and $\Gamma$ suppress $T_{\mathrm{c}}$. As expected, the size of $\Delta_{0}$ needed to destroy superconductivity is of order $T_{\mathrm{c} 0}$. In that context, it should be remarked for the experimental data used in Fig. 2, the ratio of $\Delta_{0}$ to $T_{\mathrm{c}}$ is about 6.4. The behaviour of $\lambda$ with temperature is similar to the weak-coupling case, as illustrated in Fig. 4. Again, a solution fails to appear once the temperature maximum of $\lambda$ falls below unity. The same behaviour was found in the Fermi-surface-restricted results presented in Fig. 2. In turn, use of our phenomenological $G$ and $\chi$ in the full Brillouin zone formalism leads to similar behaviour to Fig. 2 as well, with weakly temperature dependent $\lambda$ having values much less than unity (see Supplementary Information).

Over much of the doping-temperature phase diagram of the cuprates, ARPES reveals strongly lifetime-broadened features with 


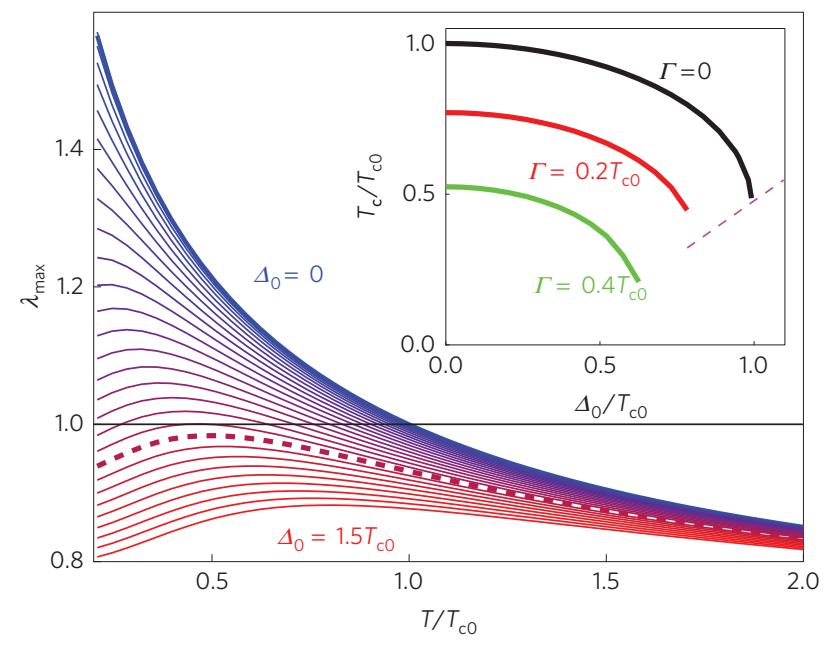

Figure 4 | Suppression of $T_{\mathrm{c}}$ by a non-pairing pseudogap (strong coupling). Inset: $d$-wave transition temperature as a function of the pseudogap $\Delta_{0}$ for various values of the inverse lifetime $\Gamma$. The energy scales are normalized to the value of $T_{\mathrm{cO}}(4.8 \mathrm{meV})$ for $\Delta_{0}, \Gamma=0$. The dashed curve is the variation of the temperature maximum in $\lambda$ for the $\Gamma=0$ case. Once the $T_{\mathrm{c}}$ curve intersects this, no solution to the superconducting gap equation exists. This is evident from the main panel, where the temperature dependence of $\lambda_{\max }$ is plotted for various $\Delta_{0}$ from 0 to $1.5 T_{\mathrm{c} 0}$. For the dashed curve and below, no solution exists.

a large pseudogap above $T_{c}$. Despite this, $T_{c}$ is large except under extreme underdoping conditions. The work presented above indicates that for such a large pseudogap, there should be no superconducting solution. In our phenomenological studies, this can be partially mitigated by using model Green's functions ${ }^{18}$ that have Fermi surfaces in the pseudogap phase (as occurs with charge ordering, spin ordering, or more phenomenological considerations such as those of ref. 23). On the other hand, the fact that we find this same behaviour using experimental Green's functions indicates that this is a general issue, not specific to any particular model.

There is a way out of this dilemma. If the pseudogap were due to pairing, then all of the above conclusions would be invalidated. In this case, the mean field $T_{c}$ would actually be the temperature at which $\Delta_{0}$ becomes non-zero (that is, $T^{*}$ ), with the true $T_{c}$ suppressed from this owing to fluctuations. In a preformed pairs picture, $T_{c}$ would be controlled by the phase stiffness of the pairs ${ }^{7}$, whereas in resonating valence bond theory, it would be controlled by the coherence temperature of the doped holes ${ }^{8}$. Regardless, our results are in strong support for such models. ARPES (refs 24,25) and scanning tunnelling microscopy ${ }^{26,27}$ results are consistent with a pairing pseudogap, because the observed spectra associated with the antinodal region of the zone have a minimum at zero bias as would be expected if the gap were due to pairing (local or otherwise). This does not mean that charge and/or spin ordering does not occur in the pseudogap phase, it is just that our results are consistent with these phenomena not being responsible for the pseudogap itself.

Received 4 October 2013; accepted 17 February 2014; published online 30 March 2014

\section{References}

1. Timusk, T. \& Statt, B. The pseudogap in high-temperature superconductors: an experimental survey. Rep. Prog. Phys. 62, 61-122 (1999).

2. Norman, M. R., Pines, D. \& Kallin, C. The pseudogap: Friend or foe of high $T_{c}$ ? Adv. Phys. 54, 715-733 (2005).

3. Kaminski, A. et al. Spontaneous breaking of time reversal symmetry in the pseudogap state of a high- $T_{c}$ superconductor. Nature 416, 610-613 (2002).
4. Fauqué, B. et al. Magnetic order in the pseudogap phase of high- $T_{c}$ superconductors. Phys. Rev. Lett. 96, 197001 (2006).

5. Xia, J. et al. Polar Kerr-effect measurements of the high-temperature $\mathrm{YBa}_{2} \mathrm{Cu}_{3} \mathrm{O}_{6+x}$ superconductor: Evidence for broken symmetry near the pseudogap temperature. Phys. Rev. Lett. 100, 127002 (2008)

6. Shekhter, A. et al. Bounding the pseudogap with a line of phase transitions in $\mathrm{YBa}_{2} \mathrm{Cu}_{3} \mathrm{O}_{6+\delta}$. Nature 498, 75-77 (2013).

7. Emery, V. J. \& Kivelson, S. A. Importance of phase fluctuations in superconductors with small superfluid density. Nature 374, 434-437 (1995)

8. Lee, P. A., Nagaosa, N. \& Wen, X-G. Doping a Mott insulator: Physics of high-temperature superconductivity. Rev. Mod. Phys. 78, 17-85 (2006).

9. Chatterjee, U. et al. Dynamic spin-response function of the high-temperature $\mathrm{Bi}_{2} \mathrm{Sr}_{2} \mathrm{CaCu}_{2} \mathrm{O}_{8+\delta}$ superconductor from angle-resolved photoemission spectra. Phys. Rev. B 75, 172504 (2007).

10. Kaminski, A. et al. Renormalization of spectral line shape and dispersion below $T_{c}$ in $\mathrm{Bi}_{2} \mathrm{Sr}_{2} \mathrm{CaCu}_{2} \mathrm{O}_{8+\delta}$. Phys. Rev. Lett. 86, 1070-1073 (2001).

11. Stock, C. et al. Spin dynamics near the critical doping in weakly superconducting underdoped $\mathrm{YBa}_{2} \mathrm{Cu}_{3} \mathrm{O}_{635}\left(T_{c}=18 \mathrm{~K}\right)$. Phys. Rev. B 77, 104513 (2008)

12. Hinkov, V. et al. Spin dynamics in the pseudogap state of a high-temperature superconductor. Nature Phys. 3, 780-785 (2007).

13. Monthoux, P. \& Pines, D. Spin-fluctuation-induced superconductivity in the copper oxides: A strong coupling calculation. Phys. Rev. Lett. 69, 961-964 (1992).

14. Scalapino, D. J. A common thread: The pairing interaction for unconventional superconductors. Rev. Mod. Phys. 84, 1383-1417 (2012).

15. Vilk, Y. M. \& Tremblay, A-M. S. Non-perturbative many-body approach to the Hubbard model and single-particle pseudogap. J. Phys. I 7, 1309-1368 (1997).

16. Dahm, T. et al. Strength of the spin-fluctuation-mediated pairing interaction in a high-temperature superconductor. Nature Phys. 5, 217-221 (2009).

17. Maier, T. A., Jarrell, M. \& Scalapino, D. J. Pairing interaction in the two-dimensional Hubbard model studied with a dynamic cluster quantum Monte Carlo approximation. Phys. Rev. B 74, 094513 (2006).

18. Norman, M. R., Kanigel, A., Randeria, M., Chatterjee, U. \& Campuzano, J. C. Modeling the Fermi arc in underdoped cuprates. Phys. Rev. B 76, 174501 (2007)

19. Abramowitz, M. \& Stegun, I. A. (eds) in Handbook of Mathematical Functions 806 (Washington, US Govt Print Office, 1964) Eq. 23.1.30.

20. Abrikosov, A. A. \& Gorkov, L. P. Contribution to the theory of superconducting alloys with paramagnetic impurities. Sov. Phys. JETP 12, 1243-1253 (1961).

21. Millis, A. J. Nearly antiferromagnetic Fermi liquids: An analytic Eliashberg approach. Phys. Rev. B 45, 13047-13054 (1992).

22. Abanov, A., Chubukov, A. V. \& Schmalian, J. Quantum-critical theory of the spin-fermion model and its application to cuprates: Normal state analysis. $A d v$. Phys. 52, 119-218 (2003).

23. Yang, K-Y., Rice, T. M. \& Zhang, F-C. Phenomenological theory of the pseudogap state. Phys. Rev. B 73, 174501 (2006).

24. Kanigel, A. et al. Evidence for pairing above the transition temperature of cuprate superconductors from the electronic dispersion in the pseudogap phase. Phys. Rev. Lett. 101, 137002 (2008).

25. Yang, H-B. et al. Emergence of preformed Cooper pairs from the doped Mott insulating state in $\mathrm{Bi}_{2} \mathrm{Sr}_{2} \mathrm{CaCu}_{2} \mathrm{O}_{8+\delta}$. Nature 456, 77-80 (2008).

26. Kohsaka, Y. et al. How Cooper pairs vanish approaching the Mott insulator in $\mathrm{Bi}_{2} \mathrm{Sr}_{2} \mathrm{CaCu}_{2} \mathrm{O}_{8+\delta}$. Nature 454, 1072-1078 (2008).

27. Alldredge, J. W. et al. Evolution of the electronic excitation spectrum with strongly diminishing hole density in superconducting $\mathrm{Bi}_{2} \mathrm{Sr}_{2} \mathrm{CaCu}_{2} \mathrm{O}_{8+\delta}$. Nature Phys. 4, 319-326 (2008).

\section{Acknowledgements}

The authors thank D. Scalapino for suggesting this work, and he and A. Chubukov for several helpful discussions. Work at Argonne was supported by the Centre for Emergent Superconductivity, an Energy Frontier Research Centre funded by the US DOE, Basic Energy Sciences, under Award No. DE-AC0298CH1088.

\section{Author contributions}

V.M. and M.R.N. conceived the project, carried out the work, and wrote the paper. U.C. and J.C.C. provided the data as well as ideas for the data analysis.

\section{Additional information}

Supplementary information is available in the online version of the paper. Reprints and permissions information is available online at www.nature.com/reprints.

Correspondence and requests for materials should be addressed to M.R.N.

\section{Competing financial interests}

The authors declare no competing financial interests. 